\title{
Analyse numérique de l'interaction de la houle avec une plaque verticale
}

\author{
Javier L. LARA ${ }^{1}$, Charlotte PATRIGEON ${ }^{2}$, Iñigo J. LOSADA ${ }^{1}$, \\ Nicolas GARCIA ${ }^{3}$ \\ ${ }^{1}$ Grupo de Ingeniería Oceanográfica y de Costas (GIOC), Instituto de Hidráulica \\ Ambiental (IH Cantabria), Universidad de Cantabria, Avda de los Castros s/n, \\ 39005, Santander, Espagne.lopezjav@unican.es, losadai@unican.es \\ ${ }^{2}$ Ecole Nationale des Ponts et Chaussées, 6 et 8 Avenue Blaise Pascal, Champs- \\ sur-Marne, 77455 Marne-la-Vallée, France.patrigec@eleves.enpc.fr \\ ${ }^{3}$ SOGREAH Maritime, 6, rue de Lorraine, 38130 Echirolles, France. \\ nicolas.garcia@sogreah.fr
}

\section{Résumé :}

L'interaction de la houle avec une plaque verticale est analysée à l'aide d'un canal à houle numérique, appelé COBRAS-UC (LOSADA et al., 2008). L'étude consiste en la validation du modèle numérique à partir de mesures disponibles dans la littérature d'agitation au voisinage de la plaque, de run-up et d'efforts sur la plaque, pour différentes hauteurs d'eau et conditions de houle incidente. La concordance obtenue entre données numériques et expérimentales est bonne. Le modèle simule avec précision les grandeurs essentielles au dimensionnement de la plaque : réflexion, transmission, efforts induits par la houle.

\begin{abstract}
:
Wave interaction with vertical barriers is modelled and investigated using the COBRAS-UC numerical flume (LOSADA et al., 2008). The study consists of the validation of the numerical model based on published experimental data of wave motion at both sides of the barrier, run-up and forces on the barrier, for different water depths and wave conditions. The numerical data compare well with the measured values. The numerical model is shown to correctly reproduce the main magnitudes of interest for the design: wave reflection, transmission and forces.
\end{abstract}

\section{Mots-clés :}

Interaction houle-structure, modèle numérique, plaque verticale, onde solitaire, houle monochromatique, houle irrégulière, efforts, dimensionnement. 


\section{$1 \quad$ Introduction}

On entend par plaque verticale un mur vertical imperméable émergent, avec une longueur de pénétration dans l'eau (ou tirant d'eau) variable, utilisé en général pour réduire la transmission des ondes courtes dans un bassin portuaire. Un schéma de principe est montré Figure 1. L'interaction de la houle régulière et/ou irrégulière avec ce type d'ouvrages a fait l'objet d'un certain nombre de travaux de modélisation théorique, physique et plus récemment numérique, parmi lesquels WIEGEL (1960), KRIEBEL (1992), LOSADA et al. (1994), KRIEBEL et al. (1998), GILMAN \& KRIEBEL (1999), LIU \& AL-BANAA (2004). Le travail présenté ici consiste en la validation et l'exploitation du modèle numérique COBRAS-UC développé par le Groupe d'Ingénierie Océanographique et Côtière de l'Université de Cantabrie (Espagne) à des fins de dimensionnement, fonctionnel et structurel, des plaques verticales. Ce travail de validation utilise les données expérimentales publiées par LIU \& AL-BANAA (2004) et KRIEBEL et al. (1998), décrites plus loin. L'effort de validation porte sur les cas de complexité croissante d'onde solitaire, de houle monochromatique et de houle aléatoire. Les grandeurs étudiées sont les suivantes : l'évolution de la surface libre et le champ des vitesses au voisinage de la plaque; la réflexion, la transmission et la dissipation de la houle induites par la plaque ; le run-up sur la plaque ; les efforts induits par la houle sur la plaque.

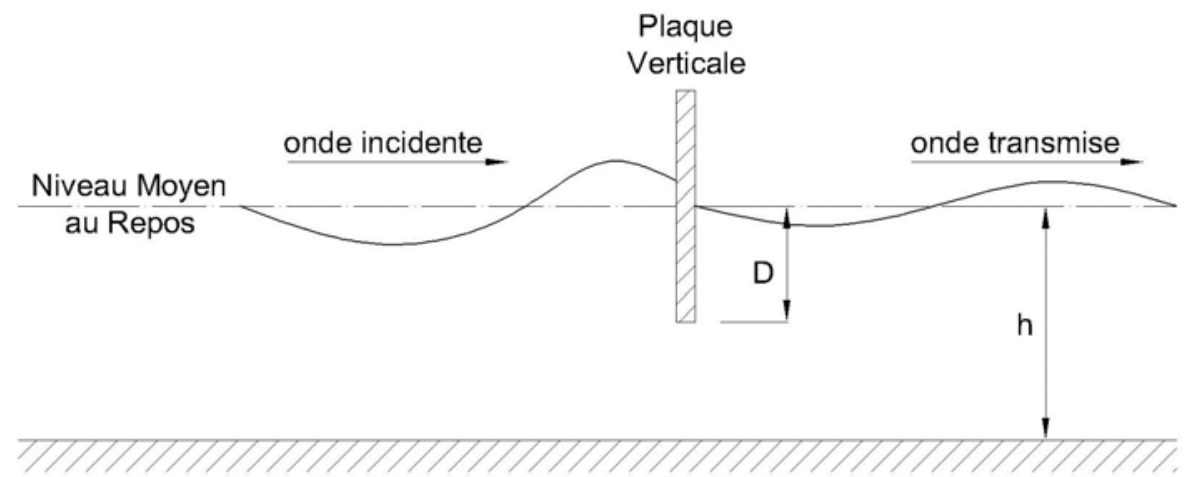

Figure 1. Schéma de principe d'une plaque verticale.

\section{Description du modèle numérique}

L'étude est réalisée à l'aide du code bidimensionnel «COBRAS-UC », de type RANS (Reynolds-Averaged Navier Stokes), développé par l'Université de Cantabrie (Santander, Espagne) à partir du code initialement développé par Liu et al. (1999). Les équations RANS se basent sur la décomposition du champ des vitesses et des pressions instantanées en une composante moyenne et une fluctuation turbulente. Les effets de la turbulence sont représentés à l'aide d'un 
modèle k-E non linéaire. Le suivi de la surface libre est réalisé à l'aide de la méthode VOF (Volume Of Fluid), qui consiste à déterminer les variations de densité dans chacune des mailles du domaine de calcul. Le code inclut différentes procédures de génération de la houle, parmi lesquelles un mode «batteur à houle » similaire à celui des canaux à houle réels et un mode de génération interne, consistant en la définition d'une fonction source dans l'équation de continuité. Une description plus détaillée du code est fournie par Losada et al. (2008). COBRAS-UC a été validé et utilisé pour l'étude de l'interaction de la houle avec différents types d'ouvrages côtiers, comme les brise-lames à faible arase (GARCIA et al., 2004) ou les digues à talus (LOSADA et al., 2008). Ces derniers auteurs ont mis en évidence en particulier la bonne précision des valeurs numériques de franchissements et d'efforts sur le mur de garde de l'ouvrage. Le code a également été validé et utilisé récemment pour l'étude de la propagation des ondes longues sur les plages réelles (TORRES-FREYERMUTH et al., 2007).

\section{$3 \quad$ Validation du modèle numérique}

\subsection{Cas d'onde solitaire}

La première étape du travail de validation présenté ici porte sur le cas de l'interaction avec une plaque verticale d'une onde solitaire, à partir des données expérimentales présentées par LIU \& AL-BANAA (2004). Ces données sont issues de huit essais en canal à houle à petite échelle et incluent des mesures de surface libre, de run-up et d'efforts pour différentes valeurs de hauteur de l'onde incidente, de profondeur d'eau et de tirant d'eau de la plaque. Les huit essais ont été reproduits numériquement à l'aide de COBRAS-UC, en considérant un maillage non uniforme sur l'ensemble du domaine de calcul et une résolution au voisinage de la plaque de $\Delta \mathrm{x}=\Delta \mathrm{y}=1 \mathrm{~mm}$. Une comparaison des séries temporelles de déformée de surface libre devant et derrière la plaque, pour une profondeur d'eau de $11 \mathrm{~cm}$, une onde incidente de $3.4 \mathrm{~cm}$ et un tirant d'eau de la plaque de $6.2 \mathrm{~cm}$, est présentée Figure 1. La figure illustre le bon niveau de performance du code. Le biais sur la hauteur de l'onde principale côté mer est d'environ $3 \%$, et d'environ $20 \%$ sur la hauteur des deux ondes suivantes (on notera que ces ondes secondaires présentent des hauteurs très faibles, inférieure à $15 \mathrm{~mm}$ ). La transmission de l'énergie incidente est également précisément représentée. Le biais sur la hauteur de l'onde principale à l'arrière de la plaque est d'environ 3\%, sur la hauteur de l'onde secondaire de 16\%. Par souci de concision un seul cas est présenté ici, mais les autres essais sont également correctement simulés. Une analyse de sensibilité sur la résolution du domaine numérique a montré qu'un maillage plus grossier, $\Delta \mathrm{x}=8 \mathrm{~mm}$ (maillage horizontal) et 
$\Delta y=2 \mathrm{~mm}$ (maillage vertical), permettait de réduire les temps de calcul (d'environ un facteur deux) en maintenant un niveau de précision comparable sur le calcul de la hauteur de l'onde incidente (principale), nécessaire à un calcul précis du run-up sur la plaque et des efforts induits. La comparaison des résultats expérimentaux et numériques de run-up montre également une bonne concordance entre les deux types de données, avec un biais inférieur à $2 \%$ avec le maillage fin initial et à $10 \%$ avec le maillage plus grossier. Ces résultats, comme tous ceux présentés dans cette étude, ont été obtenus sans calage préalable particulier du modèle.

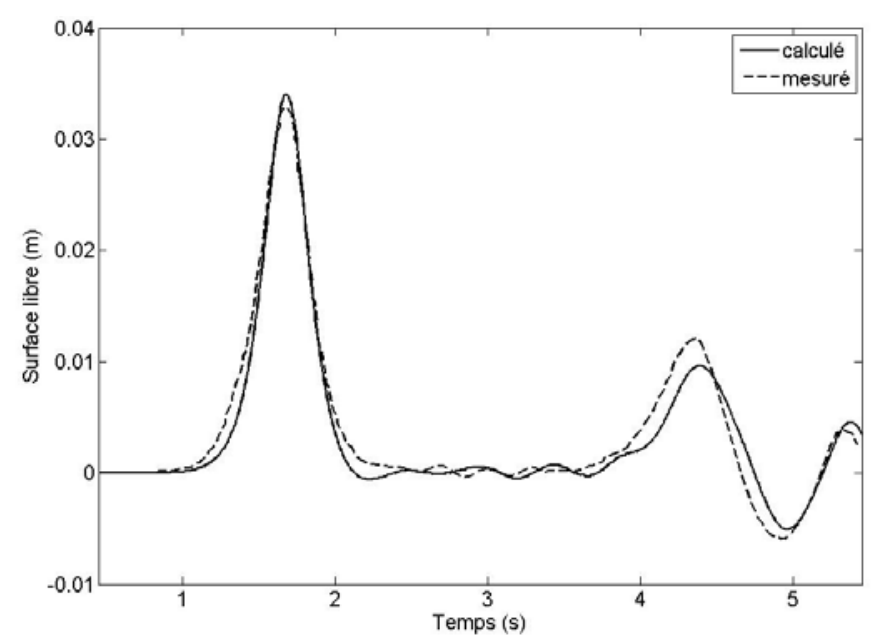

Figure 2. Evolution de la surface libre devant la plaque $(x=-1.524 \mathrm{~m})$.

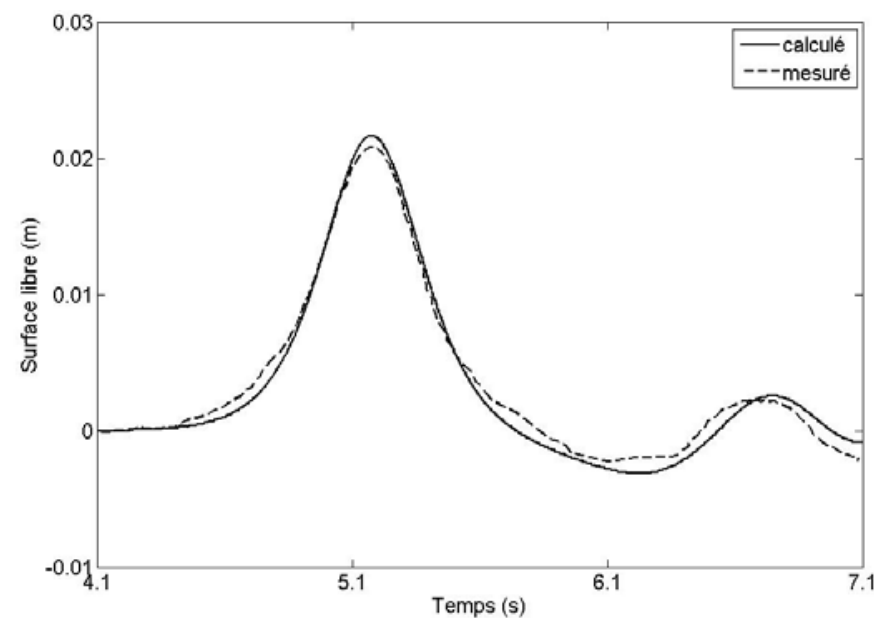

Figure 3. Evolution de la surface libre derrière la plaque $(x=+2.5 \mathrm{~m})$.

La Figure 4 présente les efforts sur la plaque mesurés et calculés pour le même cas que celui de la Figure 1. Les efforts ont été déterminés lors des essais sur modèle physique à partir de la mesure des pressions sur les deux faces de la 
plaque. Les valeurs numériques d'effort sont calculées de manière similaire par intégration des valeurs de pression sur la plaque, selon l'expression :

$F=\int_{-D}^{0} P_{u p}-P_{d o w n} d z$

Avec $P_{u p}$ et $P_{\text {down }}$ la pression dynamique sur les faces respectivement avant et arrière de la plaque. L'évolution temporelle des efforts sur la plaque est correctement représentée. Un léger décrochage en crête, non mesuré en laboratoire, est observé sur le profil obtenu numériquement, une différence qui peut s'expliquer par la sensibilité du modèle numérique à la formation du tourbillon à l'aval de la plaque. Le module des efforts est calculé avec une erreur de l'ordre de $5 \%$.

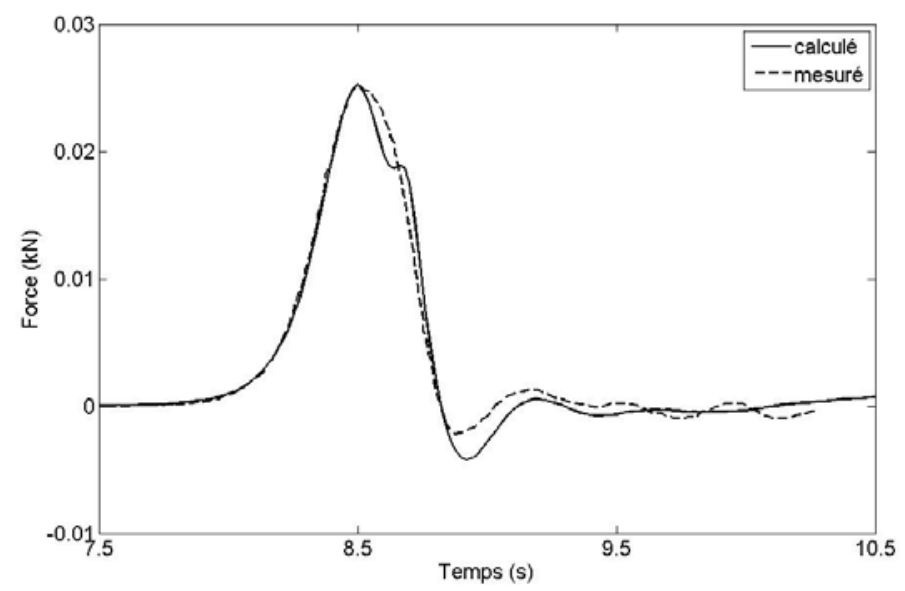

Figure 4. Evolution des efforts exercés sur la plaque.

\subsection{Cas de houle monochromatique}

Des simulations d'essais sur modèle physique en houle monochromatique ont également été réalisées et analysées. Les essais considérés sont ceux présentées par KRIEBEL et al. (1998), réalisés au «Naval Academy Hydromechanics Laboratory (NAHL) » et à " l'Oregon State University » (OSU), dans des canaux à houle de 37 et $104 \mathrm{~m}$ de long respectivement. Des mesures d'efforts sur la plaque pour différentes valeurs de profondeur relative $(\mathrm{h} / \mathrm{L}$ compris entre 0.1 à 0.5 , avec $\mathrm{L}$ la longueur d'onde), de tirant d'eau $(\mathrm{D} / \mathrm{h}=0.4,0.5,0.6,0.7)$ et de cambrure de la houle $(\mathrm{H} / \mathrm{L}=1 / 40$ et $1 / 30)$ sont présentées, et comparées aux valeurs théoriques obtenues à partir de la théorie de développement en fonctions propres («eigenfunction expansion theory»). Les essais OSU comportent également des essais en houle irrégulière, présentés au paragraphe 3.3. 

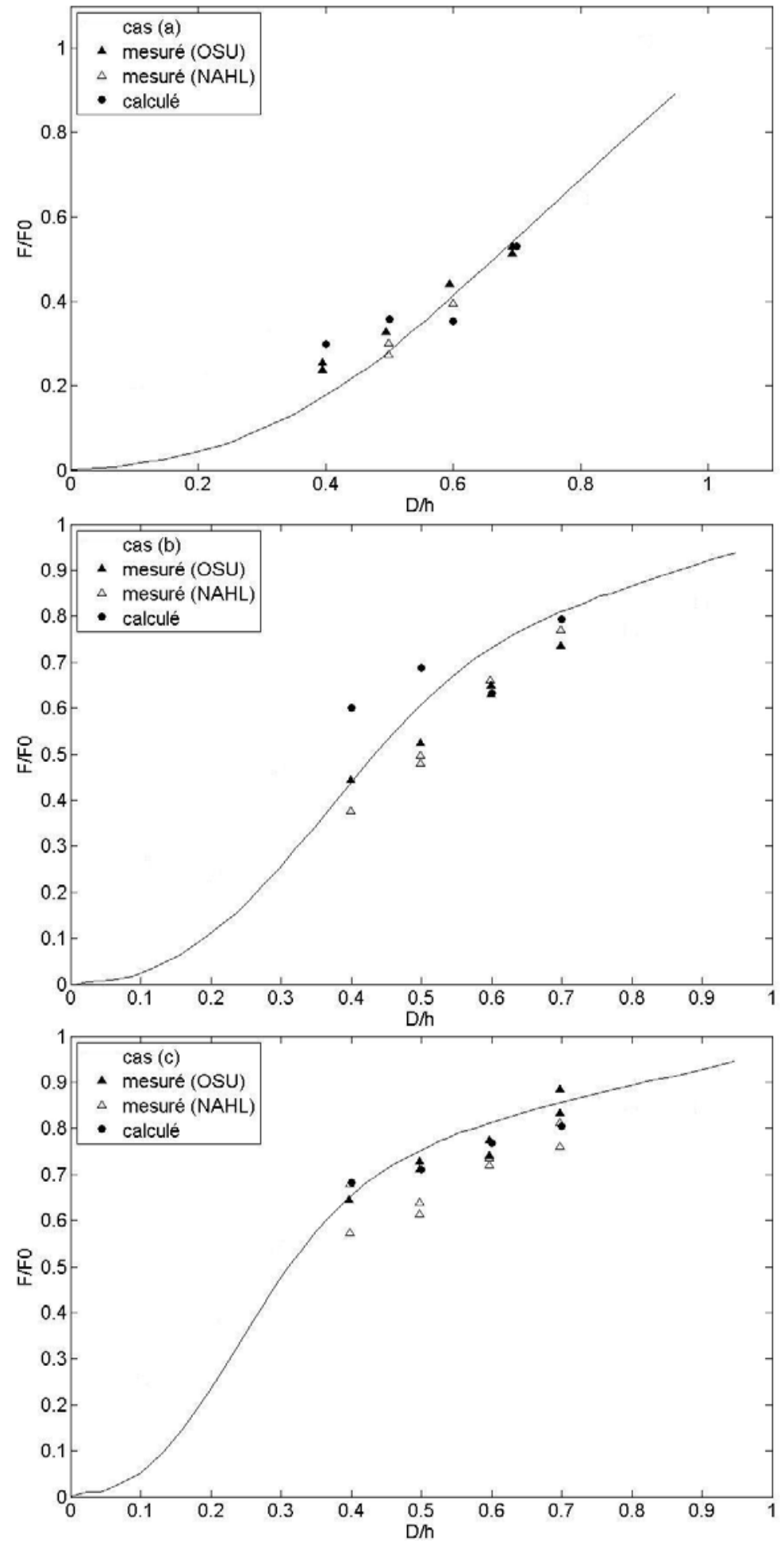

Figure 5. Comparaison des valeurs numériques et théoriques (ligne continue) d'efforts sur la plaque avec les mesures expérimentales,

(a) $h / L=0.135$, (b) $h / L=0.235$, (c) $h / L=0.335$. 
La Figure 5 montre, pour trois valeurs de profondeur relative h/L, les efforts sur la plaque mesurés en laboratoire (symboles triangulaires), évalués théoriquement (ligne continue) et calculés à l'aide du modèle numérique (symboles circulaires), en fonction du tirant d'eau $\mathrm{D}$ de la plaque rapporté à la profondeur d'eau. Ces valeurs sont rapportées à l'effort sur une plaque de tirant d'eau égal à la profondeur. Les figures illustrent l'augmentation logique des efforts sur la plaque avec son tirant d'eau, ainsi que le bon comportement du modèle numérique, à l'exception des surestimations nettes obtenues pour les faibles valeurs de $\mathrm{D} / \mathrm{h}$ pour le cas $\mathrm{h} / \mathrm{L}=0.235$. On note également le bon ajustement de la courbe théorique avec les mesures.

Comme exemple de l'exploitation des données numériques à des fins de dimensionnement fonctionnel de l'ouvrage, la Figure 6 présente l'évolution des coefficients (obtenus numériquement) de réflexion, transmission et dissipation de la houle en fonction du tirant d'eau adimensionnel $\mathrm{D} / \mathrm{h}$, pour le cas $\mathrm{h} / \mathrm{L}=0.135$. Le coefficient de réflexion est calculé à partir des séries temporelles d'évolution de la surface libre en trois points à l'amont de la plaque. On constate que ce coefficient augmente logiquement avec le tirant d'eau de la plaque alors que les coefficients de transmission et dissipation diminuent, avec une diminution plus rapide du coefficient de dissipation.

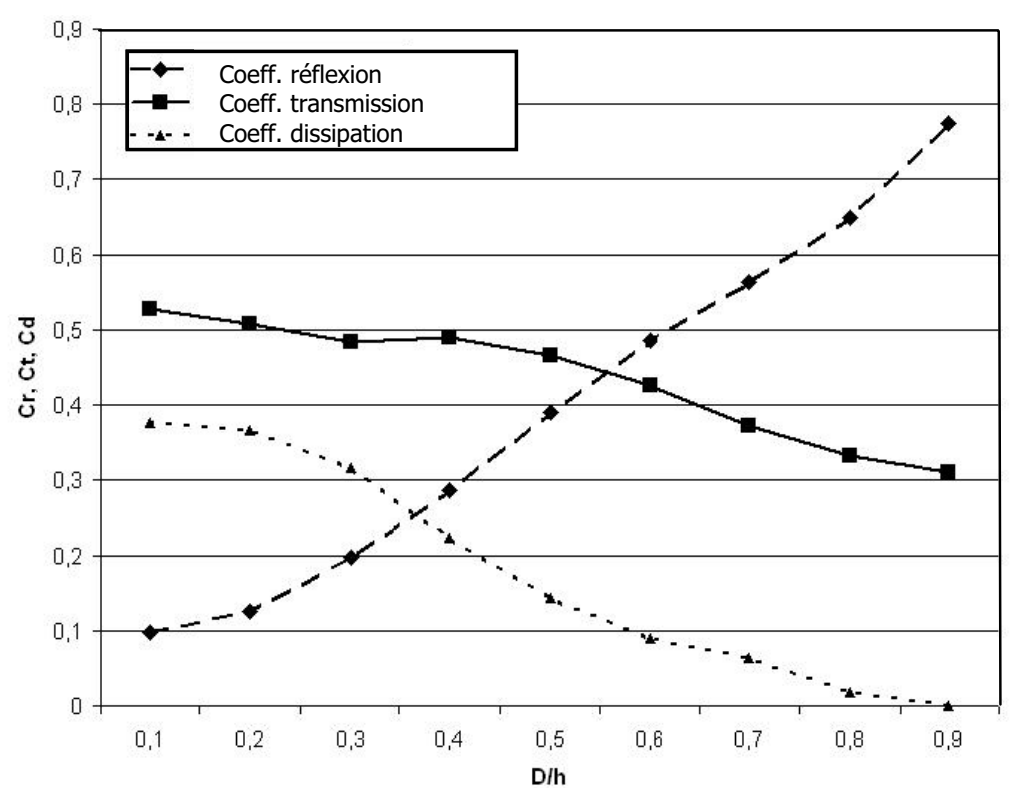

Figure 6. Evolution des coefficients de réflexion, transmission et dissipation avec le tirant d'eau de la plaque (pour le cas $h / L=0.135$ ).

Pour compléter l'analyse de la performance hydraulique de l'ouvrage, la Figure 7 présente le champ des vitesses au voisinage de la plaque à quatre instants 
pour le cas $\mathrm{h} / \mathrm{L}=0.135$ et $\mathrm{D} / \mathrm{h}=0.4$. La vitesse $(\mathrm{u})$ des particules représentée vectoriellement est adimensionnalisée avec la célérité (c) de la houle incidente. La figure illustre la structure d'écoulement autour de la plaque associé à ce cas de profondeur intermédiaire : les vitesses horizontales sont élevées et un tourbillon de grandes dimensions se forme, ce qui conduit à une transmission et une dissipation importantes de l'énergie incidente.
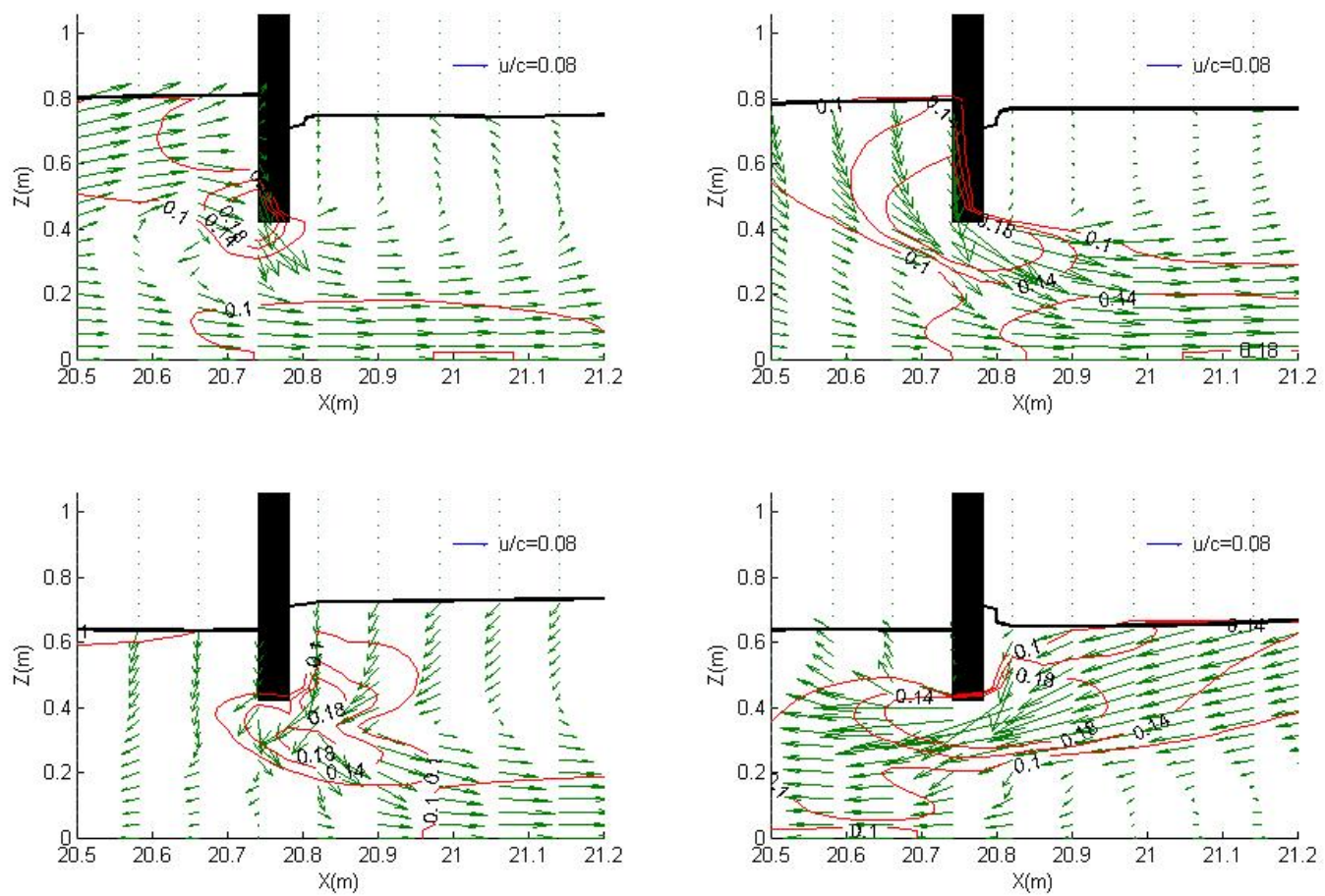

Figure 7. Champ des vitesses au voisinage de la plaque en houle monochromatique obtenu à l'aide du modèle numérique $(h / L=0.135, D / h=0.4)$.

\subsection{Cas de houle irrégulière}

KRIEBEL et al. (1988) présentent également des mesures d'efforts sur la plaque en houle irrégulière, pour plusieurs valeurs de profondeur relative $\mathrm{h} / \mathrm{L}$. Des simulations numériques du cas $\mathrm{h} / \mathrm{L}=014$ sont présentées ici, pour deux valeurs de tirant d'eau de la plaque $(\mathrm{D} / \mathrm{h}=0.4$ et 0.5$)$. Le spectre considéré est un spectre JONSWAP, $\gamma=3.3$, avec une période de pic $\mathrm{T}_{\mathrm{p}}=3.1 \mathrm{~s}$ et une hauteur de houle significative $\mathrm{H}_{\mathrm{m} 0}=35.4 \mathrm{~cm}$. La profondeur d'eau est de $1.5 \mathrm{~m}$. Le principe de la génération de la houle aléatoire dans le modèle COBRAS-UC est décrit par LARA et al. (2006). Les simulations ont été conduites sur une grille de résolution $2 \mathrm{~cm} \times 2 \mathrm{~cm}$ au voisinage de la plaque. La comparaison des valeurs d'efforts sur la plaque mesurées en laboratoire, décrites par la théorie de développement en fonctions propres et calculées à l'aide du modèle numérique est présentée 
Figure 13. Les valeurs montrées correspondent, pour les trois types de données, au moment d'ordre $0 \mathrm{du}$ spectre d'efforts sur la plaque calculé à partir de la série temporelle mesurée ou calculée. Les valeurs théoriques sont calculées selon l'expression décrite par KRIEBEL et al. (1998). Toutes les valeurs sont rapportées à la valeur d'effort pour une plaque de tirant d'eau égal à la profondeur d'eau. Les valeurs numériques obtenues pour les deux valeurs du paramètre $\mathrm{D} / \mathrm{h}$ étudiées s'ajustent très bien aux mesures correspondantes. Les valeurs théoriques s'ajustent également remarquablement bien aux données expérimentales, comme souligné par KRIEBEL et al. (1998).

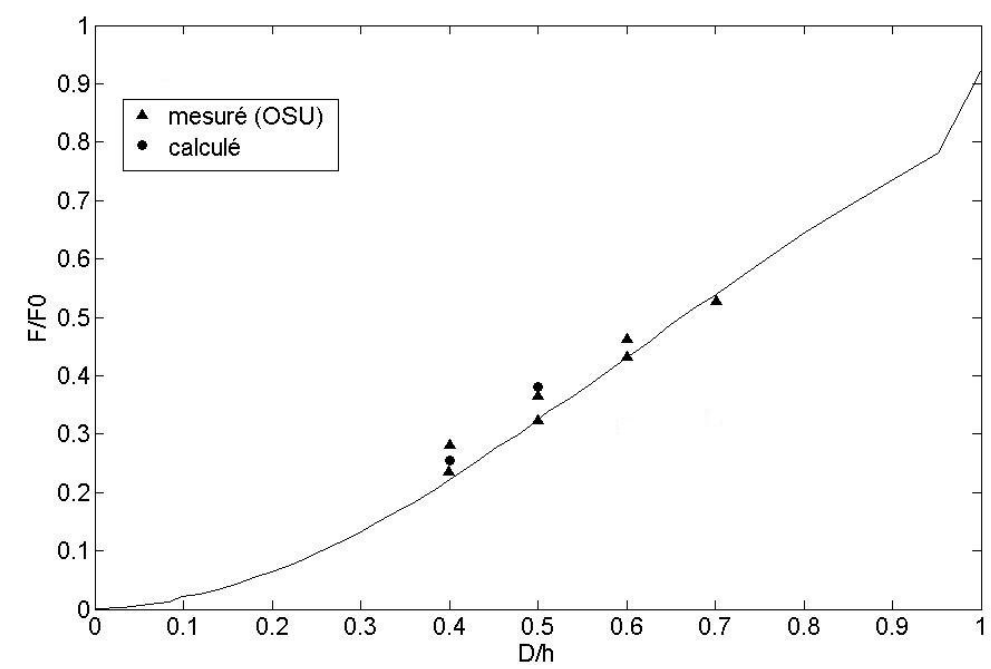

Figure 8. Efforts sur la plaque en houle irrégulière $(h / L=0.14)$. Comparaison des valeurs expérimentales, théoriques (ligne continue) et numériques.

\section{$4 \quad$ Conclusions}

Des simulations numériques de l'interaction de la houle avec une plaque verticale ont été réalisées à l'aide du modèle COBRAS-UC, de type RANS-VOF, pour des cas d'onde solitaire et de houle monochromatique et aléatoire. Des mesures d'efforts sur la plaque sont comparées aux données calculées à l'aide du modèle. Pour les cas d'onde solitaire et de houle monochromatique, l'agitation de part et d'autre de la plaque et le run-up sur la plaque sont également étudiés. Les comparaisons réalisées montrent un bon ajustement des valeurs numériques et expérimentales. Le modèle numérique reproduit correctement les phénomènes de transformation de la houle liés à la présence de la plaque, ainsi que les efforts induits sur la plaque. Les résultats préliminaires obtenus pour des cas de houle irrégulière sont prometteurs. L'effort de validation en houle irrégulière est à poursuivre pour définitivement valider l'outil numérique à des fins de dimensionnement. L'objectif final de l'étude est en effet l'utilisation et 
l'exploitation du modèle pour le dimensionnement des plaques verticales, notamment par la mise au point, à partir d'une base de données numériques (validées), d'abaques de dimensionnement fonctionnel (coefficients de réflexion/transmission/dissipation en fonction des caractéristiques de la houle incidente, de la profondeur d'eau et du tirant d'eau de la plaque) et de formules de calcul d'efforts sur la plaque.

\section{$5 \quad$ Références bibliographiques}

1 GARCIA N., LARA, J.L. LOSADA I.J. (2004). 2-D Numerical analysis of near-field flow at low-crested breakwaters. Coastal Engineering, 51 (10), 991-1020.

2 GILMAN J., KRIEBEL D. (1999). Partial depth pile supported wave barriers: a design procedure. Proceedings of Coastal Structures, 549-558. 3 KRIEBEL D. (1992). Vertical wave barriers: wave transmission and wave forces. Proceedings of the $23^{\text {rd }}$ International Conference on Coastal Engineering, 1313-1326.

4 KRIEBEL D., SOLLITT C., GERKEN W. (1998). Wave forces on a vertical wave barrier. Proceedings of the $26^{\text {th }}$ International Conference on Coastal Engineering, 2069-2081.

5 LARA J.L., GARCIA N., LOSADA I.J. (2006). RANS modelling applied to random wave interaction with submerged porous structures. Coastal Engineering, 53 (5-6), 395-417.

6 LOSADA I.J., LOSADA M., LOSADA R. (1994). Wave spectrum scattering by vertical thin barriers. Applied Ocean Research, Vol. 16, 123-128.

7 LOSADA I.J., LARA J.L., GUANCHE R., GONZALEZ-ONDINA J.M. (2008). Numerical analysis of wave overtopping of rubble mound breakwaters. Coastal Engineering, 55 (1), 47-62.

8 LIU P.F.L., AL-BANAA K. (2004). Solitary wave runup and force on a vertical barrier. Journal of Fluid Mechanics (505), 225-233. 9 LIU P.F.L., LIN P., CHANG K.A., SAKAKIYAMA T. (1999).

Numerical modeling of wave interaction with porous structures. Journal of Waterway, Port, Coastal and Ocean Engineering, ASCE, 125(6): 322-330. 10 TORRES-FREYERMUTH, A., LOSADA I.J., LARA J.L. (2007). Modeling of surf zone processes on a natural beach using ReynoldsAveraged Navier-Stokes equations. Journal of Geophysical Research, 112. 11 WIEGEL, R. (1960). Transmission of waves past a rigid vertical thin barrier. Journal of Waterways Harbors Div., ASCE 86, 1-12. 